\title{
Neural Tool Condition Estimation in The Grinding of Advanced Ceramics
}

\author{
M. E. Nakai, H. G. Junior, P. R. Aguiar, E. C. Bianchi and D. H. Spatti
}

\begin{abstract}
Ceramic parts are increasingly replacing metal parts due to their excellent physical, chemical and mechanical properties, however they also make them difficult to manufacture by traditional machining methods. The developments carried out in this work are used to estimate tool wear during the grinding of advanced ceramics. The learning process was fed with data collected from a surface grinding machine with tangential diamond wheel and alumina ceramic test specimens, in three cutting configurations: with depths of cut of $120 \mu \mathrm{m}, 70 \mu \mathrm{m}$ and $20 \mu \mathrm{m}$. The grinding wheel speed was $35 \mathrm{~m} / \mathrm{s}$ and the table speed 2.3m/s. Four neural models were evaluated, namely: Multilayer Perceptron, Radial Basis Function, Generalized Regression Neural Networks and the Adaptive Neuro-Fuzzy Inference System. The models' performance evaluation routines were executed automatically, testing all the possible combinations of inputs, number of neurons, number of layers, and spreading. The computational results reveal that the neural models were highly successful in estimating tool wear, since the errors were lower than $4 \%$.
\end{abstract}

Keywords- Ceramic grinding, RBF, GRNN, ANFIS, advanced ceramics.

\section{INTRODUÇÃO}

$\mathrm{D}$ EVIDO à baixa densidade, superior resistência ao desgaste e a corrosão e suportabilidade a altas temperaturas, as cerâmicas estruturais avançadas, tais como o óxido de alumínio, nitreto de silício e a zircônia estão sendo cada vez mais utilizados na engenharia e na medicina $[1,2]$.

Destaca-se no processo de usinagem deste tipo de cerâmica a retificação como sendo um dos estágios mais importantes, pois é de elevada complexidade e envolve o contato de grande número de partículas abrasivas com a superfície da peça [3].

A retificação também influência diretamente na rugosidade, sendo este um fator essencial na inspeção em várias aplicações industriais, como a produção e processamento de metais, semicondutores, cerâmicas, papel e plástico [4].

Estimar o desgaste das ferramentas envolvidas com o processo de usinagem tem sido uma tônica na comunidade técnico científica atualmente, pois melhorias significativas na eficiência destes processos podem ser obtidas com modelos capazes de identificar e determinar variáveis críticas que

M. E. Nakai, Departamento de Engenharia Elétrica da Faculdade de Engenharia de Bauru, UNESP, Bauru SP, Brasil, nakaimauricio@gmail.com

H. G. Junior, Departamento de Engenharia Elétrica da Faculdade de Engenharia de Bauru, UNESP, Bauru SP, Brasil, hildogjr@gmail.com

P. R. Aguiar, Departamento de Engenharia Elétrica da Faculdade de Engenharia de Bauru, UNESP, Bauru SP, Brasil, aguiarpr@feb.unesp.br

E. C. Bianchi, Departamento de Engenharia Mecânica da Faculdade de Engenharia de Bauru, UNESP, Bauru SP, Brasil, bianchi@feb.unesp.br

D. H. Spatti, Departamento de Engenharia Elétrica da Faculdade de Engenharia de Bauru, UNESP, Bauru SP, Brasil, danilospatti@gmail.com conduzem a uma resposta desejada [5,6].

No caso do desgaste do rebolo, pontuam-se três fases: uma alta taxa de desgaste inicial, seguida de um crescimento aproximadamente constante da taxa de desgaste e sofrendo uma aceleração elevada nesta taxa num terceiro momento [7].

Um dos modos de se diminuir a quantidade de paradas da máquina, assim como as chances de erro no processo é a estimação do desgaste através do monitoramento dos sinais provenientes do processo de retificação, técnica esta que levaram Webster, Dong e Lindsay [8] a constatar que o sinal de emissão acústica representa com confiabilidade a existência de falhas na superfície da peça e do rebolo, viabilizando sua utilização em mais aplicações.

O controle do processo de retificação é vital para aumentar o desempenho da ferramenta em termos de acabamento superficial, tolerâncias requeridas e ciclo de operações [9].

Tendo em vista uma efetiva redução de custos, juntamente com o aumento da qualidade das peças produzidas, a aplicação de sistemas mais inteligentes em ambientes industriais e, portanto, o controle dos danos causados no processo é crucial e de interesse direto de todos os setores dependentes desse processo.

A retificação automática e inteligente é utilizada por indústrias para a produção com alta qualidade de acabamento e geometria e neste cenário destaca-se a rugosidade como um dos fatores mais importantes para se avaliar a condição da peça durante o processo de acabamento[10].

A cerâmica avançada vem sendo utilizada nos últimos 50 anos em aplicações elétricas, magnéticas, eletrônicas, óticas (cerâmica funcional), térmicas (cerâmica estrutural) e em ambientes com elevadas temperaturas [11].

São características na maioria das cerâmicas, a resistência a altas temperaturas e a desgaste, alta dureza, baixa condutividade termal e elétrica e estabilidade química [12].

As cerâmicas estruturais apresentam elevada complexidade de usinagem, devido à dureza e fragilidade. Devido à sua alta fragilidade, os componentes invariavelmente sofrem danos, como trincas superficiais ou subsuperficiais durante o processo de remoção de material, resultando em perda de resistência mecânica.

Muitos defeitos como fissuras, alta rugosidade e ranhuras podem ocorrer durante o processo de sinterização da cerâmica, resultando diretamente na redução da qualidade mecânica da sua estrutura. A retificação remove essa camada defeituosa melhorando sistematicamente as propriedades da peça [13].

E como resultado da retificação de peças de cerâmicas avançadas, verificam-se diferentes tipos de danos em consequência de três mecanismos distintos de remoção de 
material: fratura intergranular e deslocamentos de grãos; micro fratura e formação de fragmentos de grão através de trincas intragranulares; e remoção de grandes porções de grãos, por meio de lascamentos devido à propagação de trincas transgranulares.

Infelizmente a aplicação industrial de cerâmicas avançadas é restrita pelas dificuldades na usinagem e custos elevados. Consequentemente, diversas frentes de trabalho podem ser destacadas em literatura somando esforços para melhorar a eficiência destes processos [14].

O desgaste total do rebolo aumenta com o tempo de corte. Ele pode ser dividido em uma diminuição de raio e em desgaste de quina do rebolo. Se o desgaste não é compensado durante a retificação por um sistema de medição adequado, pode-se ter um erro de medida ou eventualmente surgirem vibrações na superfície da peça.

Todos os tipos de desgaste ocorrem simultaneamente em maior ou menor grau. $\mathrm{O}$ desgaste por atrito envolve a perda de afiação dos grãos abrasivos e crescimento das áreas planas devido ao atrito com o material da peça. A fratura do grão abrasivo provoca a remoção de fragmentos, e a fratura do aglomerante resulta no desalojamento do grão abrasivo inteiro devido aos esforços de corte [15].

Assim, o monitoramento inteligente e controle do processo de retificação bem como a identificação da rugosidade da peça apresentam três importantes funções: (i) detectar problemas na rugosidade que pode ocorrer durante o processo de retificação, (ii) prover informação para otimizar e controlar o processo em tempo real, (iii) contribui para consolidar uma base de dados, que é necessária na determinação das melhores condições de operação.

\section{MODELOS NEURAIS}

As redes neurais artificiais (RNAs) parametrizadas linearmente podem ser expressas matematicamente como um vetor de funções básicas, tal como uma função vetorial nãolinear, cujos argumentos são pré-processados por uma função escalar [16].

\section{Perceptron Multicamadas}

Também chamadas de redes MLP (Multlayer Perceptron), são RNA's do tipo FeedForward com treinamento supervisionado, possuem muiltiplas camadas neurais. A característica principal das redes em camadas é a presença de uma ou mais camadas escondidas, as quais são responsáveis pela extração e armazenamento de conhecimentos associados a alguma aplicação específica [17].

Em redes Perceptrons multicamadas, as "tarefas" são distribuídas através das camadas. Os estímulos ou sinais são apresentados à rede na camada de entrada. A camada intermediária extrai as informações e as codificam através dos pesos sinápticos, formando assim uma representação própria do ambiente externo.

Finalmente, a camada de saída recebe os estímulos da camada intermediária, e mapeia o padrão de resposta que será a saída da rede. O processo de treinamento destas redes é realizado pelo algoritmo conhecido como BackPropagation ou Regra Delta Generalizada. O algoritmo de treinamento é realizado em dois passos: um Passo Forward onde um padrão é aplicado nas entradas da rede $\mathrm{e}$ as informações são propagadas camada a camada até as saídas, não alterando os pesos sinápticos; Passo Backward onde a partir das saídas calcula-se o erro que será propagado, de volta, objetivando o ajuste da matriz de pesos sinápticos.

As redes MLP são as mais empregadas nas soluções de problemas de engenharia, pois possuem elevada capacidade de generalização, robustez e eficiência computacional. Suas principais aplicações são em aproximar funções, reconhecer padrões, identificações e controle [18].

\section{Redes de Base Radial}

Também conhecidas por Radial Basis Function ou RBF e assim como as redes Perceptron Multicamadas essas RNA's também são empregadas para aproximação de funções e classificação de padrões em espaços com dimensão muito grande. Os Neurônios de saída executam uma combinação Linear das funções bases computadas pelos Neurônios da camada intermediária. Portanto, como nas RNA's Perceptron Multicamadas, os Neurônios escondidos formam funções bases sigmóides que são diferentes de zero em uma região infinitamente grande do espaço de entradas. $\mathrm{Na} \mathrm{RBF}$, as respostas dos Neurônios escondidos são diferentes de zero apenas dentro de uma região localizada "muito pequena".

Em relação aos problemas de classificação de padrões, as redes MLP conseguem separar padrões referentes às diversas classes por meio da combinação de hiperplanos, enquanto que nas redes RBF as curvas de separação são funções radiais. Os critérios de distâncias métricas máximas em função de determinados pontos são especificados usando-se a verificação dos dados de entrada.

Sua superioridade, portanto, é devida à utilização de algoritmos de aprendizado linear para complementar a tarefa normalmente realizada pelos algoritmos não lineares, enquanto mantém a alta precisão dos algoritmos não lineares [19].

\section{Redes de Regressão Generalizada}

A rede neural de regressão generalizada (GRNN) tem a característica de não necessitar de um algoritmo de treinamento iterativo. A rede aproxima uma função arbitraria entre os vetores de entrada e saída, obtendo a função estimada diretamente dos dados de treinamento. Com o aumento do tamanho do grupo de treinamento, a estimativa do erro se aproxima de zero, característica altamente desejada para um estimador.

A rede neural de regressão generalizada é usada na estimação de variáveis contínuas, como em técnicas de regressão padrão. A GRNN é uma variante da rede neural RBF, fundamentada na técnica estatística chamada regressão kernel. A principal vantagem da rede GRNN é o rápido aprendizado e a convergência para uma superfície de regressão 
ótima quando se tem um número grande de amostras. A GRNN tem bom desempenho com dados esparsos em um ambiente de tempo real porque a sua superfície de regressão é instantaneamente definida em qualquer local do espaço amostral [20].

A GRNN é capaz de aproximar qualquer função arbitrária, mesmo existindo relações não lineares entre as variáveis de entrada e a saída, criando a função de estimação de saída diretamente.

Esta característica é particularmente vantajosa com dados esparsos em uma aplicação em tempo real, sendo esta sua principal diferença em relação às redes mais populares, já que seu aprendizado não utiliza uma abordagem de ajuste iterativo para adquirir informação sobre o treinamento.

\section{Sistema de Inferência Neuro-Fuzzy}

Trata-se dos Sistemas Híbridos mais pesquisados na atualidade, associando duas técnicas de modelagem muito populares (Redes Neurais e a Lógica Fuzzy). Enquadra-se no tipo de sistema híbrido incorporado. Consiste em Implementar um Sistema de Inferência Fuzzy (FIS - Fuzzy Inference System) através de uma arquitetura paralela distribuída como a de uma Rede Neural, de forma que os algoritmos de aprendizado possam ser usados para ajustar o FIS.

Associam a capacidade de aprendizado e de tolerância a falhas das RNA, com a interpretabilidade dos Sistemas de Inferência Fuzzy, assim, permitem a integração de conhecimento explícito (de especialistas) e conhecimento implícito (conjunto de dados) e extração de conhecimento sob a forma de regras de inferência fuzzy. São aplicados à problemas de reconhecimento de padrões // sistemas de classificação de dados, sistemas para diagnóstico // mineração de dados, aproximação de funções // identificação e controle e previsão de séries temporais.

A estrutura básica de um sistema Neuro-Fuzzy ANFIS, também chamado fuzzy Sugeno ou fuzzy do tipo 3, se baseia na equivalência funcional, sob certas restrições, entre as redes RBF e os sistemas fuzzy do tipo TSK. Neste modelo uma única saída é calculada diretamente sem defuzzificação, como uma ponderação das entradas de acordo com as regras fuzzy, que podem ser determinadas por um algoritmo computacional baseado em redes neurais [21].

\section{Metodologia Experimental Para Coleta de Dados}

As condições de retificação e dressagem foram configuradas de acordo com as experiências adquiridas pelo grupo de Usinagem por Abrasão, sendo os principais parâmetros registrados na Tabela I. Os módulos de aquisição de emissão acústica (DM42) e de potência média foram ligados a um bloco de conectores modelo BNC2110, que por sua vez foi conectado a uma placa de aquisição, Modelo PCI$6035 \mathrm{E}$ de 16 bits de resolução e frequência máxima de aquisição de $200 \mathrm{kS} / \mathrm{s}$, fabricado pela National InstrumentsTM.

A coleta dos dados foi realizada sob a plataforma do programa computacional LabVIEW®.

TABELA I. CONFIGURAÇÃO DOS PARÂMETROS DE RETIFICAÇÃO.
\begin{tabular}{|l|c|}
\hline Velocidade de Corte $\left(v_{s}\right)$ & $35 \mathrm{~m} / \mathrm{s}$ \\
\hline Velocidade da Peça $\left(v_{w}\right)$ & $2.3 \mathrm{~m} / \mathrm{min}$. \\
\hline Vazão do Fluido & $27.51 \mathrm{I} / \mathrm{min}$. \\
\hline Pressão do Fluido & $<0.2 \mathrm{Kgf} / \mathrm{cm}^{2}$ \\
\hline Concentração do Fluido & $4 \%$ \\
\hline Profundidade de Dressagem & $2 \mu \mathrm{m}$ \\
\hline Passos de Dressagem & 8 \\
\hline
\end{tabular}

O cabo do sensor de emissão acústica foi conectado na porta de aquisição RMS, ou seja, todos os sinais coletados de emissão acústica foram valores RMS. Esta opção foi escolhida em função dos objetivos deste trabalho de usar o sinal RMS de EA como entrada para os modelos neurais juntamente com o sinal de potência de corte e o parâmetro DPO, sendo este último função dos valores RMS de EA. A frequência de aquisição foi configurada para $2 \mathrm{KS} / \mathrm{s}$.

No módulo de aquisição de potência é possível coletar sinais de corrente instantânea, corrente RMS, tensão instantânea, e potência média. O sinal monitorado foi o de potência média e a taxa de aquisição foi a mesma que a da emissão acústica, $2 \mathrm{KS} / \mathrm{s}$. A Tabela II mostra os valores ajustados no módulo de aquisição de potência.

TABELA II. CÁlCULO DA ESPESSURA EQUIVALENTE DE CORTE.

\begin{tabular}{|c|c|c|c|c|}
\hline & $\begin{array}{c}\text { Profundida } \\
\text { de corte }(\boldsymbol{a})\end{array}$ & $\begin{array}{c}\text { Velocidade } \\
\text { de Corte }\left(\boldsymbol{v}_{\boldsymbol{s}}\right)\end{array}$ & $\begin{array}{c}\text { Velocidade } \\
\text { da Mesa }\left(\boldsymbol{v}_{\boldsymbol{w}}\right)\end{array}$ & $\mathbf{h}_{\mathbf{e q}}$ \\
\hline Test 1 & $20 \mu \mathrm{m}$ & $35 \mathrm{~m} / \mathrm{s}$ & $2.3 \mathrm{~m} / \mathrm{min}$ & $0.02 \mu \mathrm{m}$ \\
\hline Test 2 & $70 \mu \mathrm{m}$ & $35 \mathrm{~m} / \mathrm{s}$ & $2.3 \mathrm{~m} / \mathrm{min}$ & $0.07 \mu \mathrm{m}$ \\
\hline Test 3 & $120 \mu \mathrm{m}$ & $35 \mathrm{~m} / \mathrm{s}$ & $2.3 \mathrm{~m} / \mathrm{min}$ & $0.132 \mu \mathrm{m}$ \\
\hline
\end{tabular}

A interface gráfica de aquisição de sinais (LabVIEW) foi preparada para coletar os sinais de potência média e emissão acústica RMS concomitantemente. Foi configurada a opção de salvar os arquivos em formato binário de 16 bits.

Após a montagem do banco de ensaios e configurações dos equipamentos foi realizada a coleta dos sinais e as medições da rugosidade e do desgaste. Foram escolhidas três profundidades de corte $(a)$ diferentes. Essas profundidades foram escolhidas adotando a metodologia de uma remoção branda de material $(a=20 \mu \mathrm{m})$, uma remoção intermediária de material $(a=70 \mu \mathrm{m})$ e uma remoção de material mais intensa $(a=120 \mu \mathrm{m})$.

Como a velocidade de corte $\left(v_{s}\right)$ e a velocidade da peça/mesa $\left(v_{w}\right)$ não variaram ao longo dos ensaios, a espessura equivalente de corte $\left(\mathrm{h}_{\mathrm{eq}}\right)$ ficou em função da profundidade de corte $(a)$.

Nos três ensaios, foi removida a mesma quantidade de material de $16,000 \mathrm{~mm}^{3}$. A variação do parâmetro $\mathrm{h}_{\mathrm{eq}}$ (devido à variação de profundidade de corte) resultou em uma variação na quantidade de passes de retificação para a remoção da mesma quantidade de material. Cada ensaio foi dividido em 10 partes com o mesmo número de passadas na peça. Antes de iniciar o ensaio e ao final de cada parte fora realizada a medição da rugosidade e do desgaste. O corpo-de-prova foi dividido em 11 pontos igualmente espaçados para se garantir os valores da rugosidade média ( $\mathrm{Ra})$, pois o valor de $\mathrm{Ra}$ deve 
permanecer constante ao longo do corpo-de-prova.

Em seguida, a mesa é acionada até que o rebolo corte a peça de cerâmica imprimindo o perfil da superfície contendo o degrau relacionado ao desgaste. Para cada ensaio, em cada passe de retificação foram coletados os sinais de emissão acústica (RMS) e potência média, e o número de sinais coletados por ensaio.

De posse dos arquivos dos sinais de emissão acústica e potência de corte dos três ensaios foi realizado o processamento digital dos sinais, sob a plataforma do programa computacional MATLAB, obtendo as estatísticas: média da emissão acústica (AE), desvio da emissão acústica $(\operatorname{std}(\mathrm{AE}))$, média da potência $(\mathrm{PW})$, desvio da potência $(\operatorname{std}(P W))$ e o DPO.

\section{Processo de Aprendizado dos Sistemas Neurais}

Para a obtenção de todas as configurações possíveis de conjuntos de entradas foi utilizado um algoritmo binário utilizando estas 5 estatísticas obtendo-se 31 possibilidades de entradas devido a ordem de permuta de mesmas variáveis não alterar o resultado da combinação.

Utilizou-se modelos neurais de apenas uma saída, portanto para cada tipo de rede neural avaliado obtiveram-se 3 modelos neurais, um para cada profundidade de corte, totalizando 12 modelos neurais.

Devido às inúmeras possibilidades de configuração do modelo MLP, o algoritmo utilizado foi o mais complexo e custoso computacionalmente, pois neste modelo foram confeccionadas redes com 1 a 3 camadas neurais, variando-se a quantidade de neurônios de 5 a 40 por camada.

A RBF tem como parâmetro de configuração o "spread" e devido às características da função de base radial os parâmetros de testes foram variados de forma exponencial visando proteger o algoritmo de testes de influências dos parâmetros de configuração. A variação ocorre de 0.003 a 19, valores obtidos a partir de ensaios preliminares.

Devido às características semelhantes entre modelos neurais GRNN e RBF, o parâmetro de configuração utilizado neste modelo neural também foi o "spread", sendo este variado também de forma exponencial: de 0.003 a 19, valores obtidos a partir de ensaios preliminares.

O parâmetro de configuração testado para os modelos ANFIS foi a quantidade de funções de pertinência utilizadas, variando-se de 2 a 9 . Testes preliminares demonstraram que quantidades de funções de pertinência maiores que 9 não resultaram na diminuição do erro médio de saída dos modelos testados.

Através do algoritmo de teste de influência dos parâmetros de configuração e de conjunto de entrada foi possível determinar as melhores entradas para cada profundidade de corte e para cada modelo neural. A Fig. 1 apresenta o resultado do algoritmo para a profundidade de corte de $120 \mu \mathrm{m}$ para o modelo neural MLP.

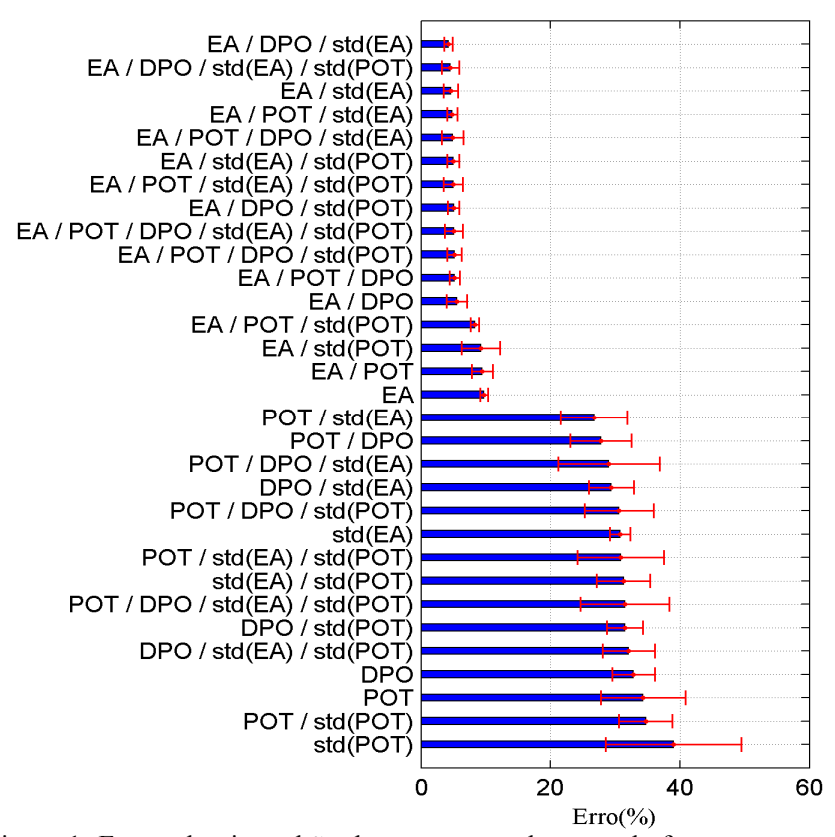

Figura 1. Erro e desvio padrão do erro para o desgaste da ferramenta para a profundidade de corte de $120 \mu \mathrm{m}$, modelo neural MLP.

Os conjuntos de entradas avaliados estão representados no eixo y e os erros médios percentuais no eixo x. Foram adotados como índice de repetibilidade da rede, os valores do desvio padrão obtido em cada rede para cada modelo neural estudado. O mesmo procedimento fora realizado para os demais modelos neurais, e podem ser conferidos nas Fig. 2, Fig. 3 e Fig. 4.

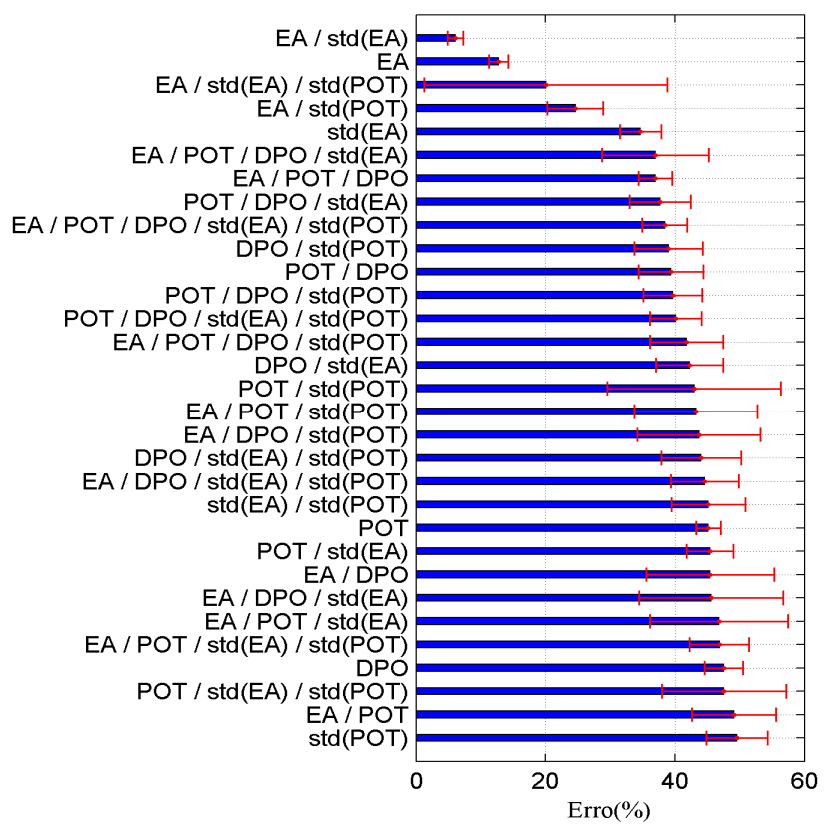

Figura 2. Erro e desvio padrão do erro para o desgaste da ferramenta para a profundidade de corte de $120 \mu \mathrm{m}$, modelo neural RBF. 


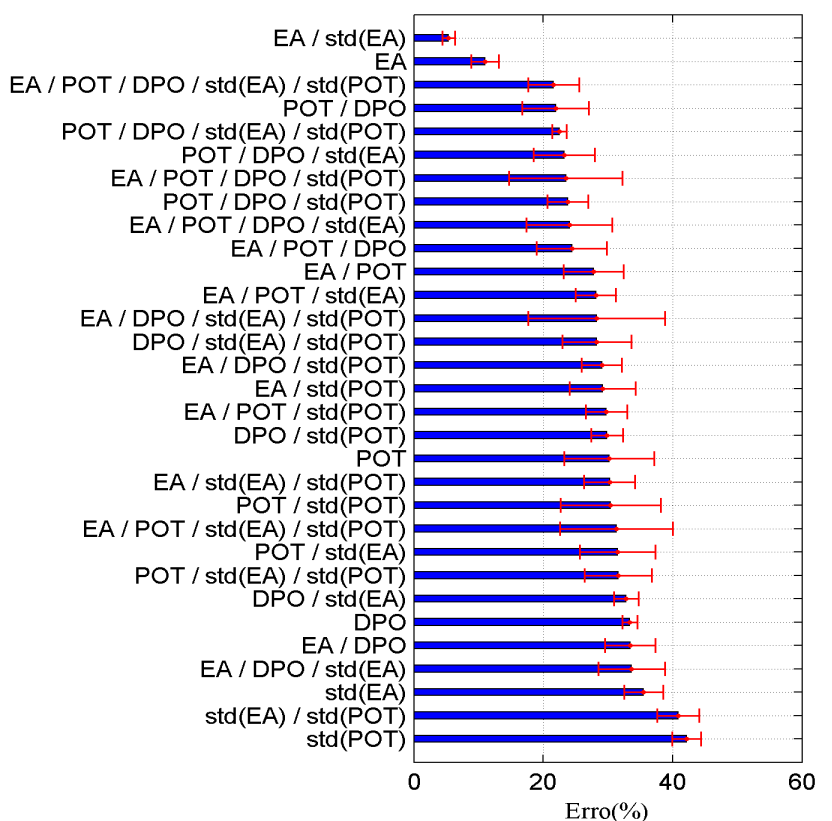

Figura 3. Erro e desvio padrão do erro para o desgaste da ferramenta para a profundidade de corte de $120 \mu \mathrm{m}$, modelo neural GRNN.

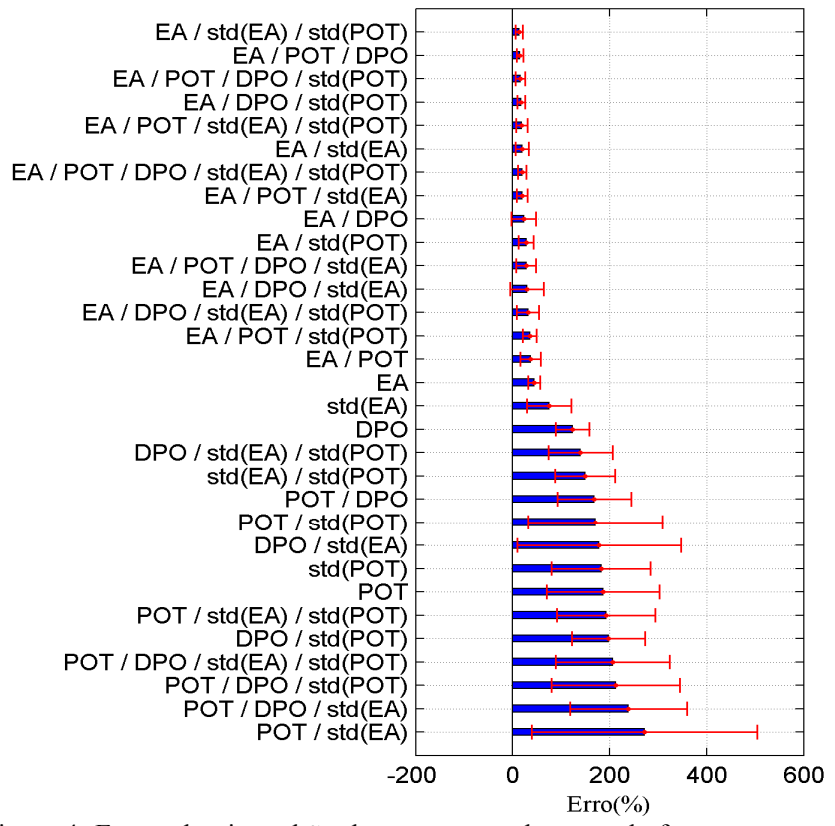

Figura 4. Erro e desvio padrão do erro para o desgaste da ferramenta para a profundidade de corte de $120 \mu \mathrm{m}$, modelo neural ANFIS.

\section{AvaliaÇão da Performance dos estimadores NEURAIS}

A Tabela III apresenta as performances resultantes do treinamento realizado com a rede neural MLP. A primeira coluna especifica a saída treinada; a segunda coluna mostra os ensaios usados no treinamento; na terceira coluna os grupos de entradas que obtiveram melhores erros percentuais; As duas colunas seguintes representam o erro percentual e desvio padrão obtidos para os modelos.

TABELA III. DESEMPENHO PARA A MELHOR REDE MLP.
\begin{tabular}{|c|c|c|c|}
\hline Saída & Entradas & Erro(\%) & Std. D. (\%) \\
\hline $120 \mu \mathrm{m}$ & AE/DPO/std(AE) & 4.190 & 0.647 \\
\hline $70 \mu \mathrm{m}$ & AE/PW/DPO/std(PW) & 4.805 & 0.969 \\
\hline $20 \mu \mathrm{m}$ & AE/PW/DPO/std(AE)/std(PW) & 4.037 & 0.358 \\
\hline
\end{tabular}

Verifica-se que somente o modelo neural que tem como saída o desgaste, para o ensaio de $70 \mu \mathrm{m}$, utilizou o número máximo de neurônios (40) da camada escondida, demonstrando que a faixa de variação para o número de neurônios da camada escondida para os modelos de estimação foi adequada.

Os erros percentuais do desgaste estão muito acima dos erros obtidos para a rugosidade, demonstrando a melhor adequação das redes ao comportamento da rugosidade. Observa-se que a média do sinal de EA está presente em todos os melhores modelos, ressaltando sua importância no processo de retificação.

A Tabela IV apresenta os resultados da rede neural RBF. Observa-se que o menor erro percentual para o desgaste foi do ensaio de $70 \mu \mathrm{m}(3.829 \%)$, onde se pode verificar que as estatísticas derivadas do sinal de EA contribuíram significativamente para o aprendizado da rede RBF.

A Tabela $\mathrm{V}$ apresenta os resultados para a melhor rede GRNN. A performance escolhida fora obtida para o desgaste no ensaio de $20 \mu \mathrm{m}(4,84 \%)$.

Na Tabela VI, observa-se que os menores desvios foram obtidos para a estimação do desgaste no ensaio de $70 \mu \mathrm{m}$ $(5,48 \%)$.

TABELA IV. DESEMPENHO PARA A MELHOR REDE RBF.
\begin{tabular}{|c|c|c|c|}
\hline Saída & Entradas & Erro(\%) & Std. D. (\%) \\
\hline $120 \mu \mathrm{m}$ & $\mathrm{AE} / \mathrm{std}(\mathrm{AE})$ & 6.075 & 1.194 \\
\hline $70 \mu \mathrm{m}$ & $\mathrm{AE} / \mathrm{std}(\mathrm{AE}) / \mathrm{std}(\mathrm{PW})$ & 4.874 & 0.613 \\
\hline $20 \mu \mathrm{m}$ & $\mathrm{AE} / \mathrm{std}(\mathrm{AE}) / \mathrm{std}(\mathrm{PW})$ & 3.829 & 0.220 \\
\hline
\end{tabular}

TABELA V. DESEMPENHO PARA A MELHOR REDE GRNN.

\begin{tabular}{|c|c|c|c|}
\hline \multicolumn{1}{|c|}{ Saída } & Entradas & Erro(\%) & Std. D.(\%) \\
\hline $120 \mu \mathrm{m}$ & $\mathrm{AE} / \mathrm{std}(\mathrm{AE})$ & 5.353 & 0.962 \\
\hline $70 \mu \mathrm{m}$ & $\mathrm{AE} / \mathrm{std}(\mathrm{AE})$ & 8.047 & 1.483 \\
\hline $20 \mu \mathrm{m}$ & $\mathrm{AE} / \mathrm{PW} / \mathrm{DPO} / \mathrm{std}(\mathrm{PW})$ & 4.843 & 0.697 \\
\hline
\end{tabular}

TABELA VI. DESEMPENHO PARA O MELHOR MODELO ANFIS.
\begin{tabular}{|c|c|c|c|}
\hline Saída & Entradas & Erro(\%) & Std. D.(\%) \\
\hline $120 \mu \mathrm{m}$ & $\mathrm{AE} / \mathrm{std}(\mathrm{AE}) / \mathrm{std}(\mathrm{PW})$ & 13.701 & 7.564 \\
\hline $70 \mu \mathrm{m}$ & $\mathrm{AE} / \mathrm{std}(\mathrm{AE}) / \operatorname{std}(\mathrm{PW})$ & 5.484 & 1.343 \\
\hline $20 \mu \mathrm{m}$ & $\mathrm{AE} / \mathrm{PW} / \operatorname{std}(\mathrm{AE}) / \operatorname{std}(\mathrm{PW})$ & 8.872 & 2.552 \\
\hline
\end{tabular}

Os sinais derivados do sinal de EA também contribuíram significativamente para o aprendizado da rede GRNN e do 
sistema ANFIS. Separando as redes por ensaio, verifica-se que a rede MLP obteve melhor desempenho em 3 ensaios: para o desgaste, os ensaios de $120 \mu \mathrm{m}$ e $70 \mu \mathrm{m}$. A rede RBF possuir melhor desempenho no desgaste para o ensaio de $20 \mu \mathrm{m}$.

No entanto, em vários casos os erros percentuais estão próximos, como por exemplo, as redes RBF e MLP para a estimação do desgaste nos ensaio de $70 \mu \mathrm{m}$. Portanto, a escolha para a implementação dessas redes dependerá de outros fatores, tais como o tempo de processamento, e a repetibilidade do modelo, avaliado pelo desvio padrão do erro.

Observou-se que para todos os tipos de redes estudadas, o tempo de processamento foi maior para o ensaio de $20 \mu \mathrm{m}$. Este fato pode ser explicado pelo maior número de amostras para o treinamento das redes neurais desse ensaio (860 passadas), comparado com demais ensaios.

O sistema ANFIS obteve o maior tempo médio de processamento, de 170 segundos, para o desgaste. Para o ensaio de $120 \mu \mathrm{m}$ o tempo médio de processamento foi de 31.973 segundos para o desgaste. Os menores tempos foram obtidos para a rede GRNN, com o tempo médio de 0.792 segundos para o ensaio de $120 \mu \mathrm{m}$ na estimação do desgaste.

Assim, a rede GRNN foi a que apresentou a maior velocidade de treinamento, seguida pelas redes RBF, MLP e ANFIS.

\section{CONCLUSÕES}

Os processos de fabricação voltados para a retificação tangencial plana agrupam diversas áreas de conhecimento, sendo todas elas exaustivamente investigadas na atualidade. No entanto, devido à complexidade do processo, diversidade das ferramentas e outros fatores, ainda é uma área que possui enorme potencial para novas pesquisas.

E neste contexto, a utilização de sistemas inteligentes para o monitoramento, notadamente aqueles fundamentados em Redes Neurais Artificiais, agrega melhorias no processo, possibilitando sua automação e, consequentemente, sua otimização.

Por serem pesquisas emergentes, este trabalho pontuou uma necessidade real da área, que tange sobre a comparação dos diversos modelos neurais disponíveis para estimação de rugosidade e desgaste do rebolo no processo de retificação.

As estatísticas utilizadas representaram muito bem as grandezas medidas, principalmente as estatísticas derivadas do sinal de EA. A potência não foi tão significativa devido à pequena área de contato entre a peça e o rebolo e à inércia do sistema rebolo-motor.

Verificou-se que os vários tipos de redes estudados se complementam, entretanto, a RNA mais adequada para este processo de fabricação é a rede MLP, pois apresentou os menores erros na profundidade de corte de $120 \mu \mathrm{m}$ e $70 \mu \mathrm{m}$ e o segundo menor erro para a profundidade de $20 \mu \mathrm{m}$.

Tal configuração não obteve os melhores resultados no tempo de treinamento, mas foi mais a generalista durante a validação.

\section{REFERÊNCIAS}

[1] Allor, R.L., Jahanmir, S., "Current problems and future directions for ceramic machining”. American Ceramic Society Bulletin 75, 1996.

[2] Chang, C.-W., Kuo, C.-P., "An investigation of laser-assisted machining of Al2O3 ceramics planning”. International Journal of Machine Tools and Manufacture 47, 452 - 461, 2007.

[3] Bianchi, E.C., Aguiar, P., Silva, E.J., Silva Jr, C.E., Fortulan, C., "Advanced ceramics: evaluation of the ground surface". Cerâmica 49, 174-177, 2003.

[4] Dhanasekar, B., Mohan, N.K., Bhaduri, B., Ramamoorthy, B., "Evaluation of surface roughness based on monochromatic speckle correlation using image processing". Precision Engineering 32, 196 206, 2008.

[5] Mukherjee, I.; Routroy, S., "Comparing the performance of neural networks developed by using Levenberg-Marquardt and Quasi-Newton with the gradient descent algorithm for modelling a multiple response grinding process". Expert Systems with Applications vol. 39, no. 3, p. 2397-2407, 2012.

[6] Elangovan, M.; Devasenapati, S.B.; Sakthivel, N.R.; Ramachandran, K.I., "Evaluation of expert system for condition monitoring of a single point cutting tool using principle component analysis and decision tree algorithm". Expert Systems with Applications vol. 38, no. 4, p. 4450 4459, 2011.

[7] Malkin, S., "Grinding technology: theory and applications of machining with abrasives". Society of Manufacturing Engineers, 1989.

[8] Webster, J., Dong, W., Lindsay, R., "Raw acoustic emission signal analysis of grinding process". CIRP Annals-Manufacturing Technology 45, 335-340, 1996.

[9] Liang, S.Y., Hecker, R.L., Landers, R.G., "Machining Process Monitoring and Control: The State-of-the-Art". Journal of Manufacturing Science and Engineering 126, 297-310, 2004.

[10] Samhouri, M.S., Surgenor, B.W., "Surface roughness in grinding: online prediction with adaptive neuro-fuzzy inference system". Transactions of NAMRI/SME 33, 57-64, 2005.

[11] Rahaman, M.N., "Ceramic Processing and Sintering". Materials Engineering, 2003.

[12] Samant, A.N., Dahotre, N.B., "Laser machining of structural ceramicsA review". Journal of the European Ceramic Society 29, 969-993, 2009.

[13] Fischer, H., Weiss, R., Telle, R., "Crack healing in alumina bioceramics. Dental materials". Official publication of the Academy of Dental Materials 24, 328-32, 2008.

[14] Agarwal, S., Rao, P.V., "Experimental investigation of surface/subsurface damage formation and material removal mechanisms in SiC grinding". International Journal of Machine Tools and Manufacture 48, 698 - 710, 2008.

[15] Linke, B., Klocke, F., "Temperatures and wear mechanisms in dressing of vitrified bonded grinding wheels". International Journal of Machine Tools and Manufacture 50, 552 - 558, 2010.

[16] Vargas, J.A.R., Gularte, K.H.M. ; Hemerly, E.M., "Adaptive observer design based on scaling and neural networks". IEEE Transactions on Latin America, vol. 11, no. 4, pp. 989-994, 2013.

[17] A. Goedtel; I. N. Silva; P.J.A. Serni; M. Suetake; C.F. Nascimento; S.A. O. da Silva, "Speed Estimation for Induction Motor Using Neural Networks Method”. IEEE Transactions on Latin America, vol. 11, no.2, pp. 768-778, 2013.

[18] Staelin, W., "Models for Neural Spike Computation and Cognition", 1 edn, CreateSpace, 2011.

[19] Ding, S., Jia, W., Su, C., Chen, J., "Research of neural network algorithm based on FA and RBF". Computer Engineering and Technology (ICCET), 2010 2nd International Conference On. pp. V7$228,2010$.

[20] Chen, T. C.; Yu, C. H. Motion control with deadzone estimation and compensation using GRNN for TWUSM drive system. Expert Systems with Applications, v. 36, n. 8, p. 10931-10941, 2009.

[21] Jang, J.-S.R., "ANFIS: adaptive-network-based fuzzy inference system". Systems, Man and Cybernetics, IEEE Transactions on 23, $665-685$, 1993. 


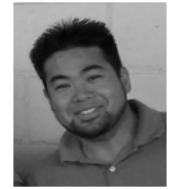

Maurício Eiji Nakai nasceu em São Paulo - SP - possui graduação em engenharia elétrica pela Universidade Estadual Paulista Julio de Mesquita Filho (UNESP - Bauru) em 2006. Mestre em ciências na área de inteligência artificial e redes neurais também pela UNESP - Bauru em 2012. Atualmente é doutorando em engenharia elétrica na área de sistemas dinâmicos e teoria de controle na área de robótica pela Universidade de São Paulo (USP - São Carlos).

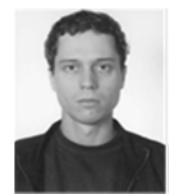

Hildo Guillardi Júnior Possui graduação em Engenharia Elétrica pela Universidade Estadual Paulista Júlio de Mesquita Filho Campus de Bauru-SP (2011) e mestrado em Engenharia Elétrica pela Universidade Estadual Paulista Júlio de Mesquita Filho Campus Bauru-SP (2013). Atualmente realiza pesquisas na área de Eletrônica de Potência, Acionamento de Máquinas Elétricas e Qualidade da Energia

Elétrica

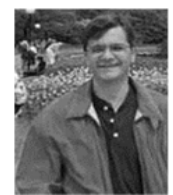

Paulo Roberto de Aguiar é Professor Titular do Departamento de Engenharia Elétrica da UNESP Bauru. Realizou programa de Pós-Doutorado na University of Connecticut, USA, na área de Processos de Fabricação. Mestre em Engenharia Elétrica pela USP São Carlos e doutor em Engenharia Elétrica pela USP São Paulo. Livre-Docência pela UNESP Bauru nas áreas de Sistemas de Controle e Processos de Fabricação. Formado em Engenharia Elétrica pela Universidade de Bauru (atual FEB-Unesp). Atualmente é coordenador do Programa de PósGraduação em Engenharia Elétrica da Faculdade de Engenharia de Bauru, UNESP.

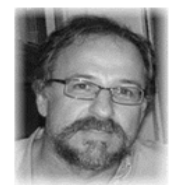

Eduardo Carlos Bianchi é Professor Titular do Departamento de Engenharia Mecânica UNESP Bauru. Mestre e doutor em Engenharia Mecânica pela USP São Carlos. Livre Docente pela UNESP Bauru na área de Engenharia Mecânica. Formado em Engenharia Mecânica e Engenharia de Segurança do Trabalho pela UNESP Bauru. Abrasão. Atualmente coordena o Grupo de Pesquisas em Usinagem por

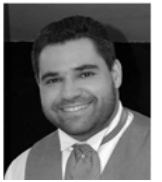

Danilo Hernane Spatti formou-se em 2005 em Engenharia Elétrica pela UNESP Bauru. No início de 2007 obteve o título de Mestre em Engenharia Elétrica pela USP São Carlos Em 2011 obteve o título de Doutor em Ciências pelo Programa de Engenharia Elétrica da EESC/USP. Atualmente é bolsista de Pós Doutorado (CAPES/PNPD) pela USP São Carlos. 